\title{
La campagne Caddiwa dans la région des îles du Cap-Vert ${ }^{1}$
}

Au cours de l'été boréal, les systèmes convectifs de méso-échelle générés au-dessus de l'Afrique de l'Ouest se propagent vers l'ouest et interagissent avec les ondes tropicales atmosphériques, et particulièrement les ondes d'est africaines, ainsi qu'avec les panaches d'aérosols terrigènes provenant du Sahel et du Sahara. Une fois au large de l'Afrique de l'Ouest, les perturbations dans le sillage de ces systèmes convectifs évoluent dans un environnement côtier complexe conduisant parfois au développement de tempêtes tropicales et d'ouragans, particulièrement en septembre lorsque les températures de surface de la mer sont élevées dans la région de l'Atlantique nord tropical et du Cap-Vert. Ces événements ont un impact significatif sur le temps en aval, depuis les Caraïbes jusqu'au États-Unis et l'Europe occidentale.

Les prévisions de la cyclogenèse dans la région du Cap-Vert sont aujourd'hui très difficiles en raison de la mauvaise compréhension des interactions « systèmes convectifs de méso-échelle-poussières terrigènes-ondes tropicales » qui limite l'horizon de prévisibilité des modèles de prévision numérique du temps. En effet, l'Afrique tropicale est la région du globe où l'on observe le plus grand nombre de systèmes convectifs de méso-échelle, dont la prise en compte constitue un défi pour les schémas de convection. En outre, les modèles actuels de prévision numérique du temps peinent à représenter le couplage entre les ondes tropicales et le flux atmosphérique moyen. Enfin, il semble que les interactions des ondes d'est africaines ainsi que d'autres ondes tropicales avec les poussières minérales, le rayonnement et les systèmes convectifs induisent des effets non linéaires dans le développement des ondes, qui sont particulièrement difficiles à prendre en compte dans les modèles de prévision numérique du temps actuels.

\section{Objectifs de la campagne de terrain}

L'objectif premier du projet CloudsAtmospheric Dynamics-Dust Interactions in West Africa (Caddiwa) est d'étudier les interactions "systèmes convectifs de méso-échelle-pousièresondes tropicales » dans la zone de l'Atlantique Nord tropical située au large de l'Afrique de l'Ouest en se basant sur des observations de pointe au sol, aéroportées et spatiales, ainsi que sur des simulations numériques régionales. Un deuxième objectif est de contribuer à la validation et à la préparation de plusieurs missions spatiales d'intérêt pour le Centre national d'études spatiales français (Cnes) et l'Agence spatiale européenne (ESA), avec en particulier la validation des missions lidar vent Aeolus, ainsi que celle de l'interféromètre atmosphérique de sondage infrarouge (Iasi) sur $M e t O p-C$ (validation) et la préparation des futures missions radar Wivern (dynamique des nuages et des précipitations) et radar-lidar EarthCare (impact radiatif des nuages et des aérosols). Un troisième objectif concerne la vérification et l'amélioration des modèles numériques de prévision régionaux.

Les questions scientifiques posées dans le cadre du projet Caddiwa sont centrées sur la compréhension des effets radiatifs liés aux aérosols terrigènes, à savoir les effets radiatifs direct, semi-direct et indirect, ainsi que sur la dynamique atmosphérique et les systèmes convectifs de méso-échelle dans l'environnement côtier complexe au large du Sénégal. Plus particulièrement, il s'agit de comprendre et d'estimer les contributions respectives des trois effets radiatifs liés aux aérosols terrigènes sur l'intensification des perturbations cycloniques de sillage des systèmes convectifs de méso-échelle au large et, éventuellement, leur transformation en tempêtes tropicales au-dessus de l'océan Atlantique nord tropical.

\section{Mise en ceuvre de la campagne}

De nouvelles observations pertinentes pour l'évaluation des interactions « systèmes convectifs de méso-échelle-poussières-ondes » ont été recueillies lors d'une campagne de terrain organisée du 6 au 23 septembre 2021 dans l'environnement tropical de l'île de Sal au Cap-Vert, située à $600 \mathrm{~km}$ au large du Sénégal. La campagne aéroportée Caddiwa, basée sur le déploiement du Falcon 20 de Safire depuis l'aéroport international Amilcar Cabral, fait partie d'un consortium expérimental international qui a opéré sous l'égide de la Joint Aeolus Tropical Atlantic Campaign (Jatac). Les mesures de la campagne Caddiwa ont été coordonnées avec

1. In memoriam, Pierre H. Flamant (21 avril 1942-30 juin 2020), qui a été l'instigateur de nombreuses missions spatiales dont la mission Aeolus. Il devait participer à la campagne Caddiwa.

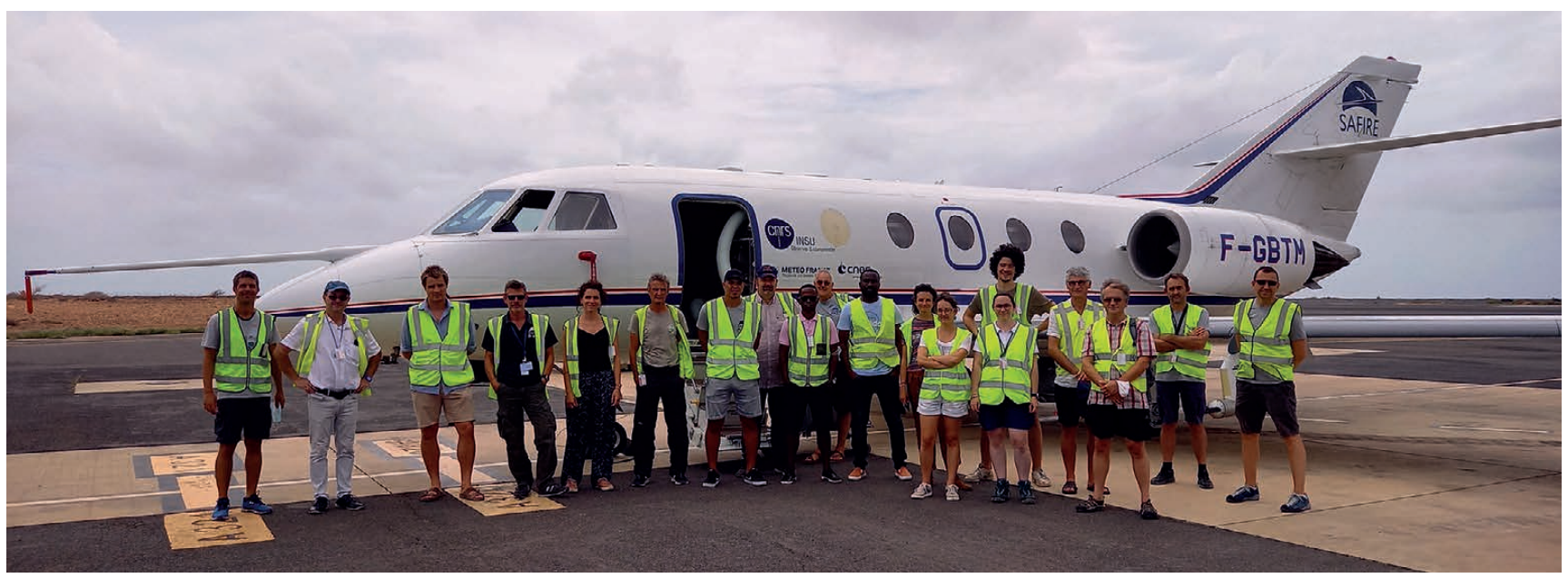

Figure 1. L'équipe Caddiwa devant le Falcon de Safire sur le tarmac de l'aéroport international Amilcar Cabral de Sal, aux îles du Cap-Vert. 
le déploiement de trois autres avions (le Falcon 20 du DLR, le WT-10 de Aerovizija Advantic et le DC8 de la Nasa), ainsi qu'avec un super site au sol mis en œuvre spécifiquement à Mindelo sur l'île de São Vicente par Tropos (Allemagne) et le NOA (Grèce). Le Falcon 20 de Safire a été équipé d'une charge utile unique dans le paysage de Jatac, réunissant l'expertise instrumentale du Latmos, du Lamp, du Lisa, de la DT-Insu et de Safire. Le Falcon 20 embarquait le système Rali (radar Rasta \& lidar Léandre nouvelle génération (LNG)), des radiomètres visible et infrarouge, des dropsondes, des sondes dynamique/thermodynamique in situ et des sondes microphysiques pour la caractérisation des aérosols et des nuages. Une station GNSS (Géolocalisation et navigation par un système de satellites) pour la mesure du contenu intégré de vapeur d'eau a également été installée dans les locaux de l'Institut national de météorologie et de géophysique (INMG) du Cap-Vert à Espargos, Sal.

En complément de ces observations et des prévisions opérationnelles globales fournies par des centres météorologiques européens, des prévisions régionales numériques ont été faites au cours de la campagne à l'aide des modèles Meso-NH et WRF-Chimere, incluant une paramétrisation de l'interaction aérosol-nuage récemment développée, afin de guider les opérations avion et de contextualiser les observations aéroportées. Ces opérations ont été portées par le Laero et le LMD. De plus, de nombreux produits aérosols, nuages et vents provenant de missions spatiales telles que les satellites Aeolus, Calipso et les instruments Iasi, Seviri et Modis ont été utilisés pendant la campagne pour permettre la contextualisation des observations acquises pendant Caddiwa. En particulier, les produits aérosols 3D Iasi développés au Lisa ont été utilisés pour cartographier la répartition verticale des panaches d'aérosols terrigènes transportés jusqu'à la zone d'étude.

Les opérations de Caddiwa ont été coordonnées depuis le centre opérationnel de Caddiwa (Cadoc) basé sur l'île de Sal, du 6 au 22 septembre 2021. Le Cadoc a conduit un briefing chaque jour à $10 \mathrm{~h}$ afin de passer en revue la situation météorologique du moment ainsi que les prévisions à 3-4 jours. En cas de mission aéroportée le lendemain, un point spécifique sur l'évolution de la situation météorologique

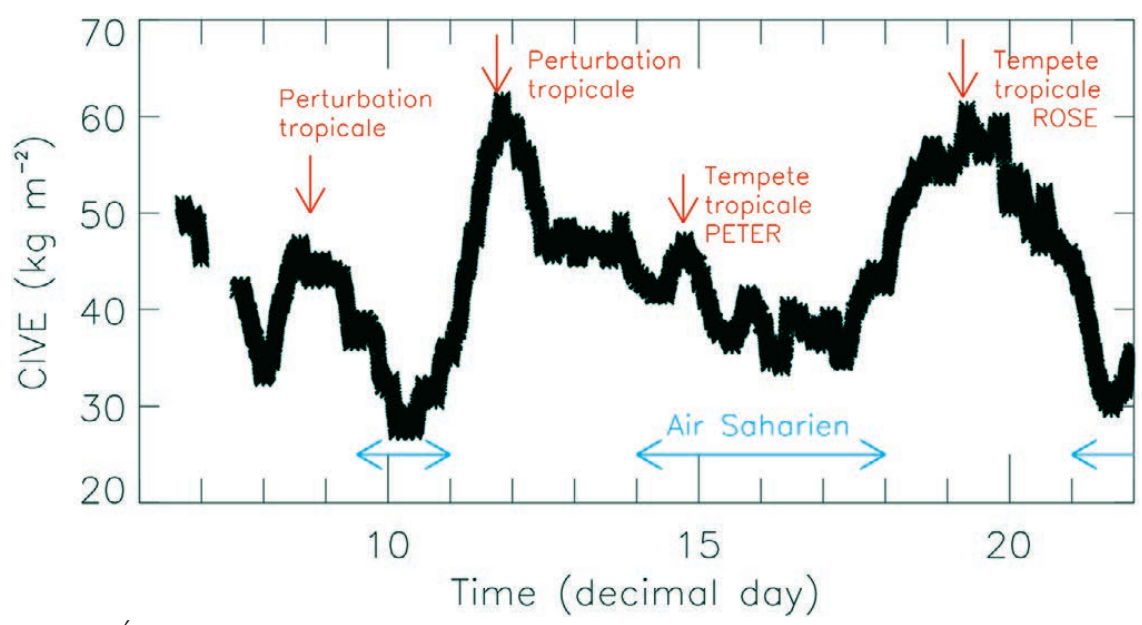

Figure 2. Évolution du contenu intégré en vapeur d'eau déduit des observations de la station GNSS installée à l'INMG à Sal, au cours de la période 6-22 septembre 2021, et identification des principaux événements météorologiques : perturbation tropicale, dépressions tropicales, transport d'air saharien.

était également programmé à $18 \mathrm{~h}$. Le groupe de prévisionnistes de la campagne a bénéficié des produits définis spécifiquement pour Caddiwa, mais également des diverses prévisions disponibles via les différentes composantes du consortium Jatac.

En tout, jusqu'à 23 personnes ont participé à la campagne, dont 7 prévisionnistes et 10 instrumentalistes. L'équipe Caddiwa (presque au complet) apparaît sur la figure 1. Des campagnes telles que Caddiwa sont lourdes à organiser, particulièrement lorsqu'il s'agit
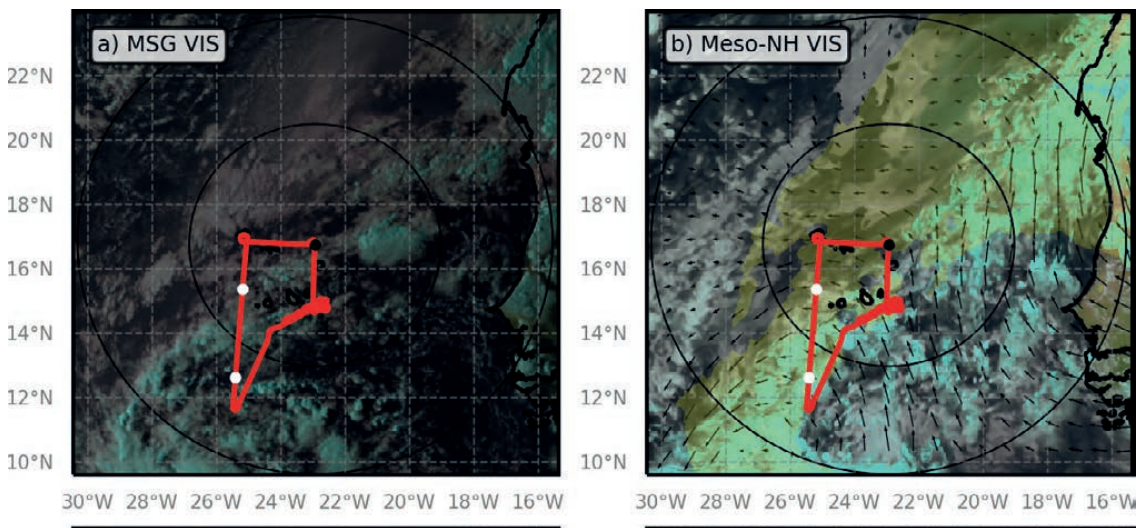

$30^{\circ} \mathrm{W} 28^{\circ} \mathrm{W} 26^{\circ} \mathrm{W} 24^{\circ} \mathrm{W} 22^{\circ} \mathrm{W} 20^{\circ} \mathrm{W} 18^{\circ} \mathrm{W} 16^{\circ} \mathrm{W}$
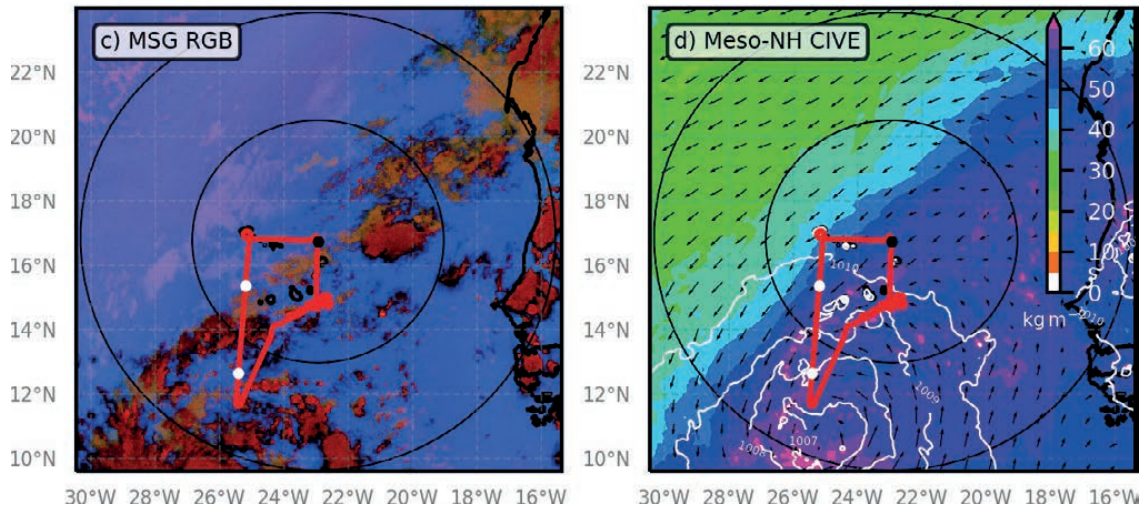

Figure 3. Situation météorologique du samedi 18 septembre 2021 à 18 h TU associée à la perturbation tropicale qui deviendra la dépression tropicale Rose deux jours plus tard. (a) Réflectance MSG dans le visible. (b) Réflectance synthétique dans le visible, issue de la prévision du modèle Meso-NH et épaisseur optique des aérosols $(>0,1)$ en jaune. Les vents à $4 \mathrm{~km}$ d'altitude sont également indiqués (flèches). (c) Image RGB MSG montrant les nuages hauts (rouge), les nuages d'étage moyen (marron foncé) et nuages bas (marron clair), ainsi que les aérosols terrigènes (rose). (d) Contenu intégré en vapeur d'eau (Cive) en couleur, pression au niveau de la mer (contour blanc) et vent à $10 \mathrm{~m}$ (flèches). La trace du vol du Falcon 20 est indiquée en rouge sur l'ensemble des figures, les points blancs localisent les dropsondes lancées depuis l'avion et le point noir indique l'aéroport sur l'île de Sal. Les deux cercles concentriques repèrent des distances de $225 \mathrm{Nm}$ et $425 \mathrm{Nm}$ à partir de Sal. 
de campagnes conduites en Afrique, et qui n'ont lieu que tous les 4 à 5 ans. La participation à une campagne internationale telle que Caddiwa est une opportunité unique pour des étudiants en master deuxième année (M2) et des doctorants s'intéressant à l'expérimentation de terrain et aux sciences environnementales, dans une région clé du globe pour la compréhension de la variabilité et du changement climatique. À ce titre, deux doctorants et deux étudiants de M2 (dont un Capverdien) ont été invités à participer à la campagne.

\section{Bilan de la campagne}

D'un point de vue climatologique, entre trois et cinq ondes d'est africaines étaient attendues au cours des trois premières semaines de septembre. Pendant la campagne Caddiwa, quatre ondes d'est africaines sont effectivement passées au sud du Cap-Vert entre le 6 et le 21 septembre, dont deux qui ont conduit à des événements météorologiques qui ont affecté l'île de Sal directement : une perturbation tropicale les 11 et 12 septembre associée à la deuxième onde de la période et la tempête tropicale Rose les 18 et 19 septembre, associée à la quatrième onde de la période. Ces événements sont visibles sur la série temporelle de contenu intégré en vapeur d'eau (figure 2). Ces deux événements ont conduit à observer les valeurs les plus élevées de la période 6-21 septembre, autour de $60 \mathrm{~kg} \mathrm{~m}^{-2}$. Les signatures de deux autres événements plus distants de Sal ont également été observées sur cette même série temporelle, avec une perturbation tropicale le 8 septembre associée à la première onde de la période et la tempête tropicale Peter les 14 et 15 septembre, associée à la troisième onde de la période. Trois épisodes de transport d'air saharien ont également affecté la zone des îles du Cap-Vert, le premier les 9 et 10 septembre, le deuxième du 14 au 18 septembre et le dernier à partir du 21 septembre. Ces événements sont également visibles sur la série temporelle de contenu intégré en vapeur d'eau comme étant associés à des conditions atmosphériques nettement plus sèches (de l'ordre de $30 \mathrm{~kg} \mathrm{~m}^{-2}$ le 10 septembre).

Neuf vols ont été réalisés avec le Falcon 20 de Safire au cours de la campagne Caddiwa, pour un total de 32 heures avec le largage de 46 dropsondes, permettant de couvrir des objectifs variés, incluant :

- l'analyse de l'environnement atmosphérique en amont du passage d'une dépression tropicale le 11 septembre lors d'un double vol, en lien avec la circulation cyclonique associée à une onde d'est africaine ;

- l'analyse de l'environnement en amont du passage de la tempête tropicale Rose les 18 et 19 septembre ;

- la validation des produits L2 (vent et aérosols) du satellite Aeolus le long d'orbites passant à proximité de l'île de Sal (deux traces descendantes le matin après $7 \mathrm{~h} \mathrm{TU}$ les 8 et 14 septembre et trois traces descendantes du soir après $19 \mathrm{~h} \mathrm{TU}$ les 10,16 et 17 septembre), grâce notamment aux mesures du lidar

(a)
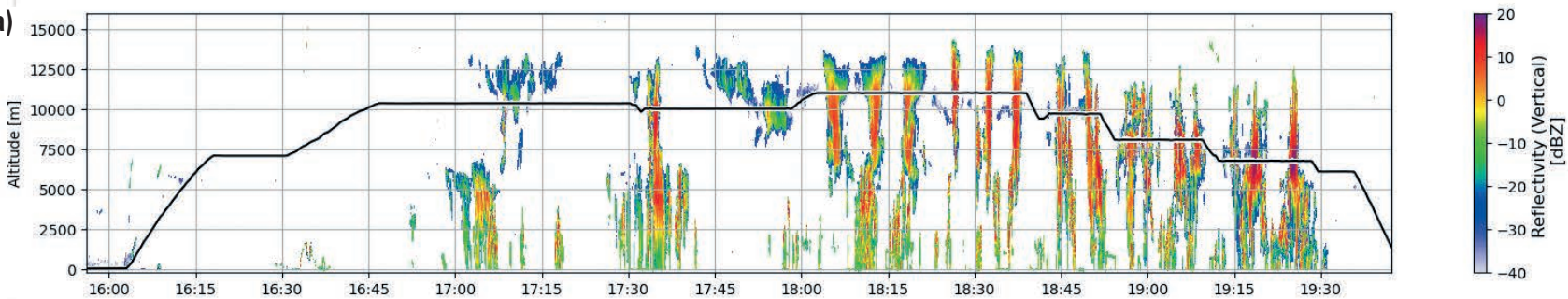

(b)
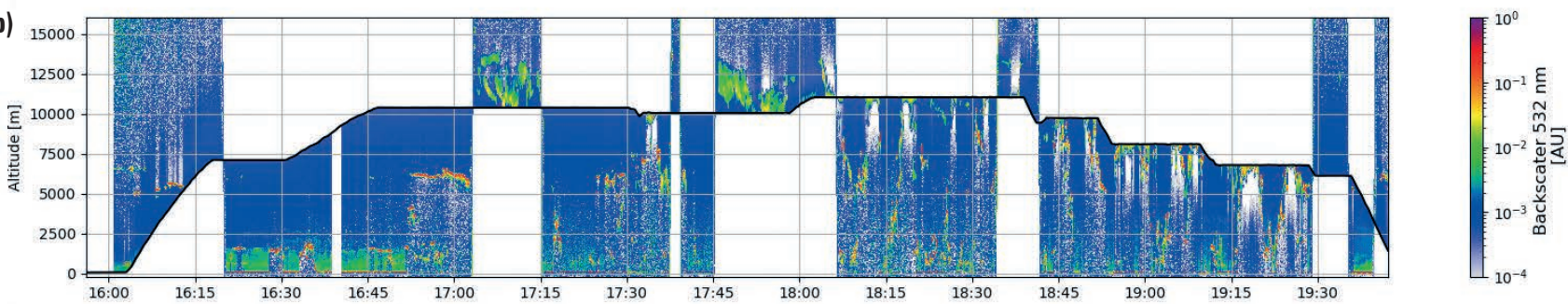

(c)
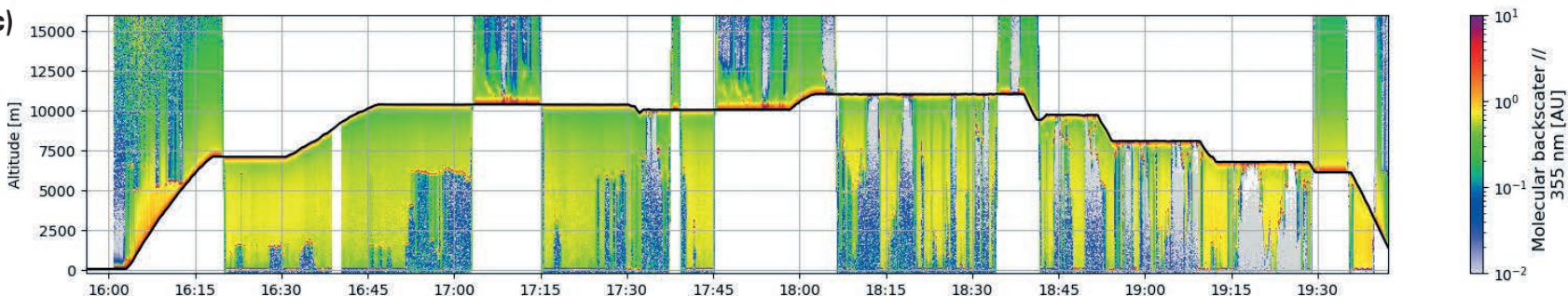

(d)
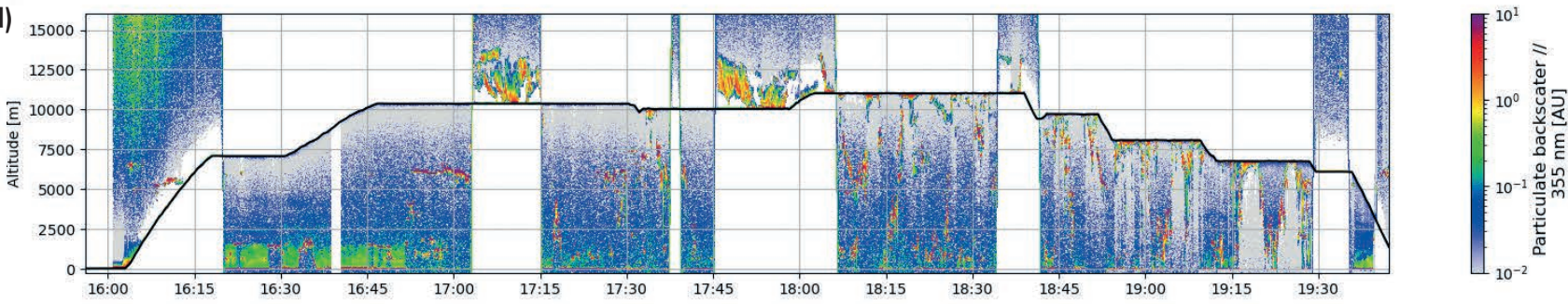

Figure 4. Profils (a) de réflectivité du radar doppler Rasta, ainsi que de rétrodiffusion lidar LNG (nadir et zénith), (b) total à 532 nm, (c) moléculaire à $355 \mathrm{~nm}$ et (d) particulaire à $355 \mathrm{~nm}$ le long du second vol du Falcon 20 de Safire du 18 septembre 2021 en amont de la perturbation tropicale qui deviendra la tempête tropicale Rose deux jours plus tard. 
LNG en mode Aeolus (en visée dépointée à $37^{\circ}$ du nadir) ;

- la faisabilité du concept des observations de la mission Wivern sélectionnée pour une phase 0 dans le cadre de Earth-Explorer 11 pour la cartographie des vents, précipitations et propriétés des nuages à l'échelle globale, grâce aux observations du radar Rasta;

- la préparation des algorithmes de la mission radar-lidar EarthCare grâce aux données synergétiques du radar Rasta et du lidar LNG (Rali) ainsi que des sondes microphysiques, obtenues lors des vols faits sur des cellules convectives dans l'environnement des dépressions et tempêtes tropicales.

\section{La tempête tropicale Rose}

La tempête tropicale Rose s'est formée sur l'Atlantique Nord tropical le 19 septembre après-midi, devenant la $17^{\mathrm{e}}$ tempête nommée de la saison 2021 des ouragans dans l'Atlantique. Rose était située à environ 300 miles nautiques au sud-ouest des îles du Cap-Vert peu avant $17 \mathrm{~h}$ TU le 19 septembre. Le Falcon 20 a échantillonné l'environnement dans lequel Rose s'est développée au cours de vols réalisés le 18 septembre après-midi (figure $3)$ et le 19 septembre au matin. Le 18 septembre après-midi, le Falcon 20 a volé en amont et dans une perturbation tropicale qui allait devenir la tempête tropicale Rose deux jours plus tard. Au cours de ce vol, des observations ont pu être acquises dans une région caractérisée par une forte humidité (figure 3d) et une forte nébulosité (figure 3a, c), au plus près du centre de la perturbation (figure 3d) autour de laquelle s'enroulait un panache d'aérosols terrigènes (figure 3b). L'intérêt de combiner des observations de télédétection radar et lidar est illustré sur la figure 4 lors du vol 12 du 18 septembre. Les données acquises par Rali permettent de détailler conjointement la répartition verticale à haute résolution spatio-temporelle des couches d'aérosols, et des nuages (nuages de couche limite, nuages moyens et les cirrus), afin de mieux appréhender leurs interactions.

Les mesures aéroportées acquises pendant la campagne ont permis de documenter les situations espérées, notamment en ce qui concerne l'environnement dynamique, en aérosols et nuageux de perturbations tropicales. Elles ouvrent de vraies perspectives pour répondre aux objectifs scientifiques de Caddiwa en termes de compréhension du rôle des interactions nuages-dynamique atmosphérique-aérosols terrigènes pour le développement des tempêtes tropicales au large du Cap-Vert. Elles permettront également de mieux contraindre les simulations qui seront réalisées à l'aide des modèles régionaux, notamment pour appréhender l'impact des différents effets radiatifs des aérosols, ainsi que de valider, améliorer et préparer, dans le cadre de scènes tropicales particulièrement complexes, différents produits satellitaires afférents à des missions d'importance pour les agences spatiales européennes.

\section{Partenaires}

Partenaires français: CNRS, Cnes, Météo-France, UCA, IPGP/IGN et Ensta-Bretagne.

Partenaires étrangers : ESA, Université du Cap-Vert à Praia et INMG au CapVert, DLR, KIT et Tropos en
Allemagne, NOA en Grèce, Université de Nova Gorica en Slovénie, Scuola Universitaria Superiore IUSS de Pavie en Italie.

Laboratoires français impliqués : Laboratoire atmosphère milieux observations spatiales (Latmos/IPSL, Île-de-France), Laboratoire de météorologie dynamique (LMD/IPSL, Île-de-France), Laboratoire interuniversitaire des systèmes atmosphérique (Lisa/IPSL, Île-de-France), Laboratoire d'aérologie (Laero/OMP, Toulouse), Laboratoire de météorologie physique (Lamp/OPGC, ClermontFerrand), Institut des géosciences de l'environnement (IGE/Osug, Grenoble), Laboratoire d'océanologie et de géosciences (LOG, Wimereux), Service des avions français instrumentés pour la recherche en environnement (Safire, CNRS/Météo-France/ Cnes, Toulouse), Division technique de l'Insu (DT-Insu, Meudon), Institut de physique du globe de Paris (IPGP, Paris).

La campagne est soutenue financièrement par l'Agence spatiale européenne (ESA), le Cnes, le CNRS-Insu (programme Lefe) et l'Institut PierreSimon Laplace (IPSL).

Cyrille Flamant, Julien Delanoë Laboratoire atmosphères, milieux, observations spatiales, IPSL

Jean-Pierre Chaboureau Laboratoire d'aérologie

Christophe Lavaysse

Institut des géosciences de l'environnement Marco Gaetani

Scuola universitaria superiore IUSS de Pavie

Olivier Bock

Institut de physique du globe de Paris

Pour en savoir plus :

Site de la campagne : https://www.aeolus.esa.int/confluence/display/TC2/

Lamorthe C., Borbon A., Schwarzenboeck A., Canonici J.-C., 2016. Safire : des avions au service de la recherche en environnement. La Météorologie, 93, 30-41.

\section{Ce qu'il faut retenir du dernier rapport du $1^{\text {er }}$ groupe de travail du Giec}

Après un mois de juillet 2021 qui a vu l'Agence internationale de l'énergie nous alerter sur la hausse continue des émissions de gaz à effet de serre, l'Amérique du Nord confrontée à des méga-feux liés à une vague de chaleur inédite, l'Allemagne et la Chine soumises à des pluies diluviennes battant des records historiques, le premier groupe de travail du Giec (WG1, 234 auteurs issus de 65 pays) a publié le 9 août dernier son sixième rapport (AR6) sur les bases physiques du changement climatique. Les principales conclusions ont été recensées dans un résumé à l'intention des décideurs approuvé par les représentants des gouvernements à l'occasion de la $54^{\mathrm{e}}$ assemblée du Giec qui, en raison de la crise sanitaire internationale, s'est déroulée en ligne sur deux semaines 